\title{
Naphthoxy Bounded Ferrocenium Salts as Cationic Photoinitiators for Epoxy Photopolymerization
}

\author{
Zh. Q. Li, ${ }^{1}$ M. Li, ${ }^{1}$ G. L. Li, ${ }^{1}$ Y. Chen, ${ }^{1}$ X. N. Wang, ${ }^{2}$ and T. Wang ${ }^{1,3}$ \\ ${ }^{1}$ State Key Lab of Chemical Resource Engineering, College of Science, Beijing University of Chemical Technology, Beijing 100029, China \\ ${ }^{2}$ College of Material Engineering, Beijing Institute of Fashion Technology, Beijing 100019, China \\ ${ }^{3}$ Department of Chemistry, Beijing University of Chemical Technology, Beijing 100029, China
}

Correspondence should be addressed to T. Wang, wangtwj2000@163.com

Received 27 February 2009; Accepted 28 April 2009

Recommended by J. Zhao

To improve the absorption and the bulk of arene ligands, two naphthoxy bounded ferrocenium salts as new cationic photoinitiators, $\left(\eta^{6}-\alpha\right.$-naphthoxybenzene) $\left(\eta^{5}\right.$-cyclopentadienyl) iron hexafluorophosphate (NOFC- 1$)$ and $\left(\eta^{6}-\beta\right.$-naphthoxybenzene) $\left(\eta^{5}\right.$ cyclopentadienyl) iron hexafluorophosphate (NOFC-2), were synthesized, characterized, and studied. NOFC-1 and NOFC-2 were prepared by the reaction of nucleophilic substitution $\left(S_{N} A r\right)$ with naphthol and chlorobenzene-cyclopentadienyliron salt. Their activity as cationic photoinitiators was studied using real-time infrared spectroscopy. The results obtained showed that NOFC-1 and NOFC-2 are capable of photoinitiating the cationic polymerization of epoxy monomer directly on irradiation with longwavelength UV light $(365 \mathrm{~nm})$. Comparative studies also demonstrated that they exhibited better efficiency than cyclopentadienylFe-cymene hexafluorophosphate (I-261). When NOFC-1 and NOFC-2 were used to efficiently initiate polymerization of epoxide, both rate of polymerization and final conversion increased using benzoyl peroxide (BPO) as sensitizer. DSC studies showed that NOFC-1 and NOFC-2 photoinitiators in epoxides possess good thermal stability in the absence of light.

Copyright ( $) 2009$ Zh. Q. Li et al. This is an open access article distributed under the Creative Commons Attribution License, which permits unrestricted use, distribution, and reproduction in any medium, provided the original work is properly cited.

\section{Introduction}

The increasing commercial and technical demands of cationic photopolymerization have led to an interest in the design and synthesis of optimized photopolymerization systems [1-4]. Currently, the most commonly used photoinitiators employed for photoinitiated cationic ring-opening poymerization are triarylsulfonium and diaryliodonium salts $[5,6]$. The relatively poor absorptivity of these photoinitiators in the near UV range, however, limits their efficiency or light utilization for commonly available light sources (including medium-pressure Hg lamp) [7-11]. Accordingly, we have pursued efforts to prepare more photosensitive cationic photoinitiators and improve their spectral absorption characteristics.

Ferrocenium salts are a different class of cationic photoinitiators [12-15]. With the absorptions caused by $\mathrm{d}-\mathrm{d}$ upfield above $300 \mathrm{~nm}$, they are more efficient than most sulfonium and iodonium salts when used with high-pressure Hg lamp as light source. Cyclopentadien-Fe-cymene hex- afluorophosphate (I-261) produced by Ciba-Geigy Corporation is the only commercial photoinitiator of iron arene complexes. For its lower molar extinction coefficients in dichloromethane at $389 \mathrm{~nm}\left(\varepsilon=75 \mathrm{M}^{-1} \cdot \mathrm{cm}^{-1}\right), \mathrm{I}-261$ has not been used widely. However, the absorption of iron arene complexes can be varied over a wide range by structure changes in the ligands.

Compared with the low molecular analogues, the presence of bulky arene ligand can reduce the migration onto the film surface, which contribute to the synthesis of low-odor and nontoxic products [16, 17]. To improve the absorption above $300 \mathrm{~nm}$ and introduce a bulky substituent, two naphthoxy bounded ferrocenium salts as new cationic photoinitiators, $\left(\eta^{6}-\alpha\right.$-naphthoxybenzene) $\left(\eta^{5}\right.$ cyclopentadienyl) iron hexafluorophosphate (NOFC-1) and $\left(\eta^{6}-\beta\right.$-naphthoxybenzene) ( $\eta^{5}$-cyclopentadienyl) iron hexafluorophosphate (NOFC-2), were synthesized. With realtime infrared spectroscopy, the study of the photopolymerization of epoxides using NOFC-1 and NOFC-2 as photoinitiators has been conducted. Direct comparisons of 


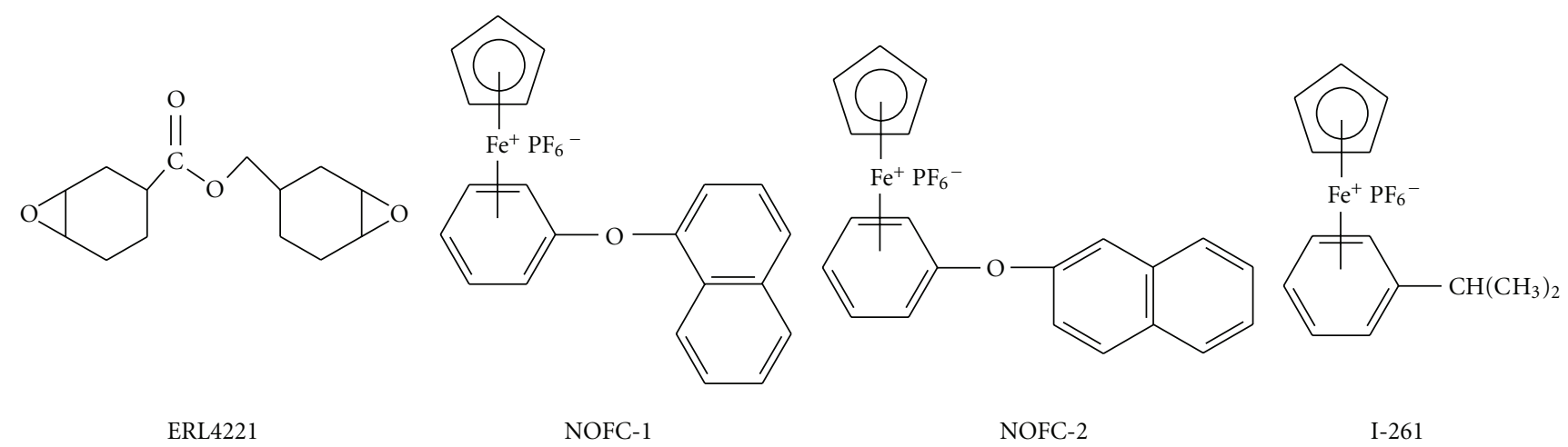

Scheme 1: Abbreviations and structures of epoxy compounds and photoinitiators employed.

these new photoinitiators with cyclopentadienyl-Fe-cymene hexafluorophosphate (I-261) are presented. Their thermal stabilities in epoxides are also studied with DSC.

\section{Experimental Section}

2.1. Materials. All starting materials used in the preparation of ferrocenium salts were reagent grade and used without purification. Epoxy compound used in this work was 3, 4epoxycyclohexylmethyl-3, 4-epoxycyclohexane carboxylate (ERL4221) which was obtained from Tianjin Synthetic Matertial Research Institute (Tianjin, China). Figure 1 summarizes the abbreviations and structures of epoxy compounds and photoinitiators used in this study.

2.2. Synthesis of NOFC-1 and NOFC-2. ( $\eta^{6}$-Chlorobenzene) $\left(\eta^{5}\right.$-cyclopentadienyl) iron hexafluorophosphate was prepared through the ligand exchange reaction between one ring of ferrocene and arene according to the reference procedure [18].

NOFC- 1 was prepared by the reaction of $\left(\eta^{6}\right.$-chlorobenzene) $\left(\eta^{5}\right.$-cyclopentadienyl) iron hexafluorophosphate with $\alpha$-naphthalenol according to the following procedure.

$\left(\eta^{6}\right.$-Chlorobenzene) $\left(\eta^{5}\right.$-cyclopentadienyl) iron hexafluorophosphate $(3.78 \mathrm{~g}, 0.01 \mathrm{~mol}), \alpha$-naphthalenol (2.88 g, $0.02 \mathrm{~mol})$ and $\mathrm{K}_{2} \mathrm{CO}_{3}(2.76 \mathrm{~g}, 0.02 \mathrm{~mol})$ were stirred in $30 \mathrm{~mL}$ of $\mathrm{N}, \mathrm{N}$-dimethylformamide (DMF) in a $100 \mathrm{~mL}$ round bottom flask under nitrogen atmosphere for 9 hours at room temperature. The reaction mixture was transferred into a $15 \%(\mathrm{v} / \mathrm{v}) \mathrm{HCl}$ solution, and a granular precipitate was formed. The obtained filtrate was washed by Acetone resulting into the dissolution of the product. This solution was then concentrated by evaporating acetone under reduced pressure, and treated with sufficient $\mathrm{NaPF}_{6}$ in water to give complete precipitation of NOFC-1 as a yellow granular solid (Yield 85\%). ${ }^{1} \mathrm{HNMR}\left(\mathrm{C}_{3} \mathrm{D}_{6} \mathrm{O}\right.$, TMS) $\delta / \mathrm{ppm}: 5.3(\mathrm{~s}, 5 \mathrm{H}, \mathrm{Cp}-\mathrm{H}), 6.3-6.5(\mathrm{~m}, 5 \mathrm{H}$, Ar-H), 7.58.1 (m, 7H, Ar-H); IR (KBr):1220(C-O-C), 829s (-PF6); m.p: $188-189^{\circ} \mathrm{C}$.

NOFC-2 was prepared by the reaction of $\left(\eta^{6}\right.$-chlorobenzene) $\left(\eta^{5}\right.$-cyclopentadienyl) iron hexafluorophosphate with $\beta$-naphthalenol in a manner similar to NOFC-1 preparation. ${ }^{1} \mathrm{H}$ NMR $\left(\mathrm{C}_{3} \mathrm{D}_{6} \mathrm{O}\right.$, TMS) $\delta / \mathrm{ppm}: 5.3$ (s, 5H, Cp-H), 6.3-6.5 (m, 5H, Ar-H), 7.5-8.1 (m, 7H, Ar-H) IR (KBr): 1250(C-O-C), 829s (PF6); m.p:180-181 ${ }^{\circ} \mathrm{C}$.

2.3. Photopolymerization Studies Using Real-Time Infrared (RTIR) Spectroscopy. The basic principle of RTIR spectroscopy consists in exposing the sample simultaneously to the UV light, which induces the polymerization, and to the infrared beam which serves to measure the monomer concentration at any given time. The resulting decrease in the IR absorption band characteristic of that monomer is monitored continuously on a transient memory recorder. Since the absorbance change is always proportional to the amount of monomer that has polymerized after a given exposure, and thus to the degree of conversion, the recorded RTIR trace actually corresponds to the conversion versus time curve.

In this paper, conversion data were obtained by monitoring the decay of the epoxy groups centered at $780-810 \mathrm{~cm}^{-1}$ (CHO) during irradiation. The mixture of monomer and photoinitiator was applied between two $\mathrm{KBr}$ crystals and irradiated with the UV radiation with UV spot light source (Rolence-100 UV, Taiwan, China) at room temperature. The light intensity on the surface of samples was $80-85 \mathrm{~mW} / \mathrm{cm}^{2}$, for each sample, the series RTIR runs were repeated three times.

2.4. Differential Scanning Calorimetric (DSC) Studies of Thermal Stability of Ferrocenium Salts. DSC studies of the thermal stability of ferrocenium salts were carried out with a Perkin Elmer Pyris 1 differential scanning calorimeter. Measurements were carried out by heating solutions of epoxide monomer containing $1.0 \mathrm{w} \%$ of ferrocenium salts in $\mathrm{N}_{2}$ at a rate of $10^{\circ} \mathrm{C} / \mathrm{min}$. The onset of the exothermic polymerization of the epoxide monomer served as a probe for the practical limit of the thermal stability of the photoinitiator.

2.5. Instruments. The ${ }^{1} \mathrm{HNMR}$ spectra were recorded on a Bruker AV600 unity spectrometer operated at $600 \mathrm{MHz}$ 

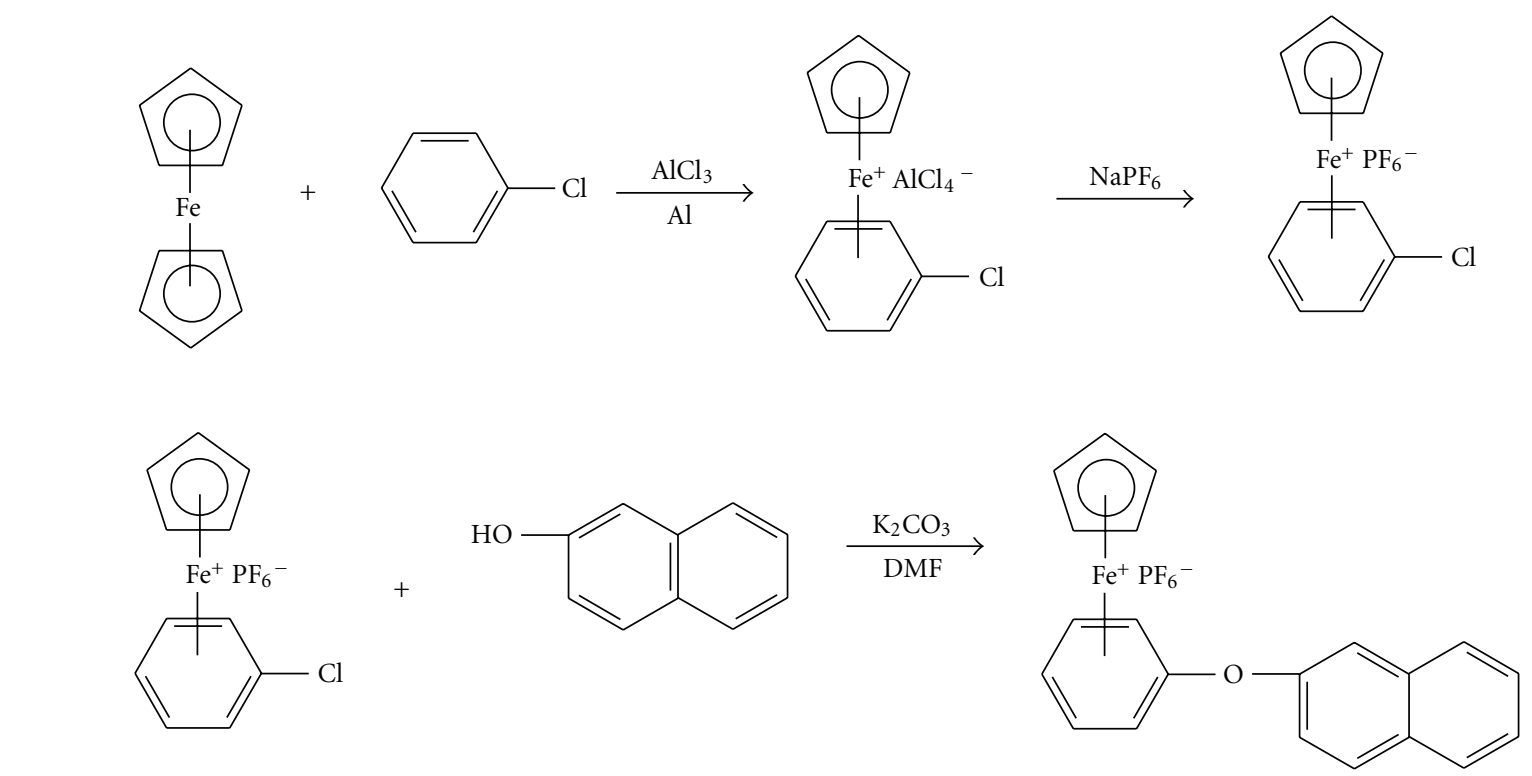

Scheme 2: Synthesis of NOFC-2.

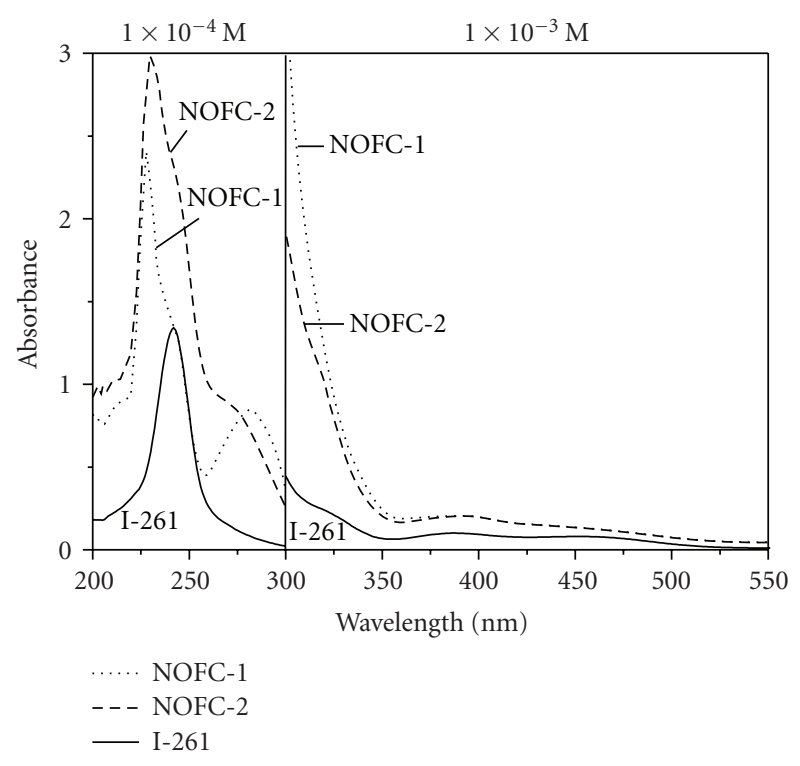

FIGURE 1: UV-Vis absorption spectra of NOFC-1, NOFC-2, and I261 in $\mathrm{CH}_{3} \mathrm{CN}$ solution.

using $\mathrm{CD}_{3} \mathrm{COCD}_{3}$ as deuterated solvent. FTIR spectra were recorded on a Nicolet 5700 instrument (Thermo Electron Corporation, Waltham, Mass, USA). UV-Vis Absorption spectra were recorded in $\mathrm{CH}_{2} \mathrm{Cl}_{2}$ solution on a Hitachi U-3010 UV-Vis spectrophotometer (Hitachi HighTechnologies Corporation,Tokyo, Japan). Light intensity was recorded by the UV light radiometer (Photoelectric Instrument Factory, Beijing Normal University, Beijing, China). DSC thermograms were measured using dynamic DSC analysis (Perkin-Elmer Pyris 1).

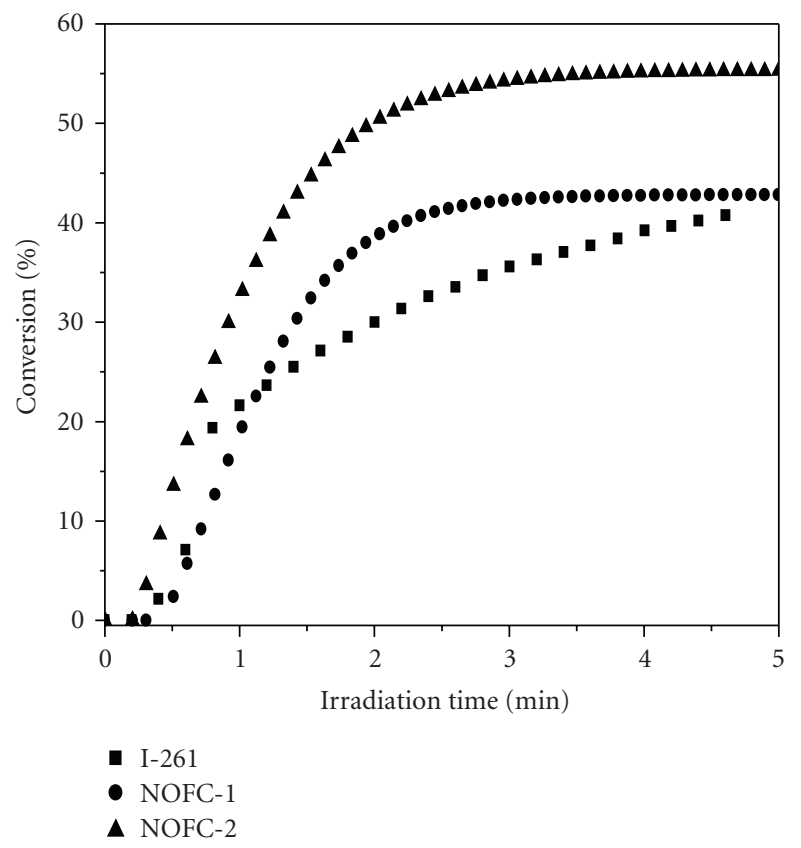

Figure 2: Conversion versus irradiation time plot of epoxy monomer ERL4221 initiated by $3.0 \mathrm{wt} \%$ photoinitiator $(\mathrm{I}=$ $\left.80 \mathrm{~mW} / \mathrm{cm}^{2}\right)$.

\section{Results and Discussion}

3.1. Synthesis of Ferrocenium Salt Photoinitiators. NOFC1 and NOFC-2 were prepared by the reaction of nucleophilic substitution $\left(\mathrm{S}_{\mathrm{N}} \mathrm{Ar}\right)$ with naphthol and chlorobenzene-cyclopentadienyliron salt. Chlorobenzenecyclopentadienyliron salt was prepared through the ligand 


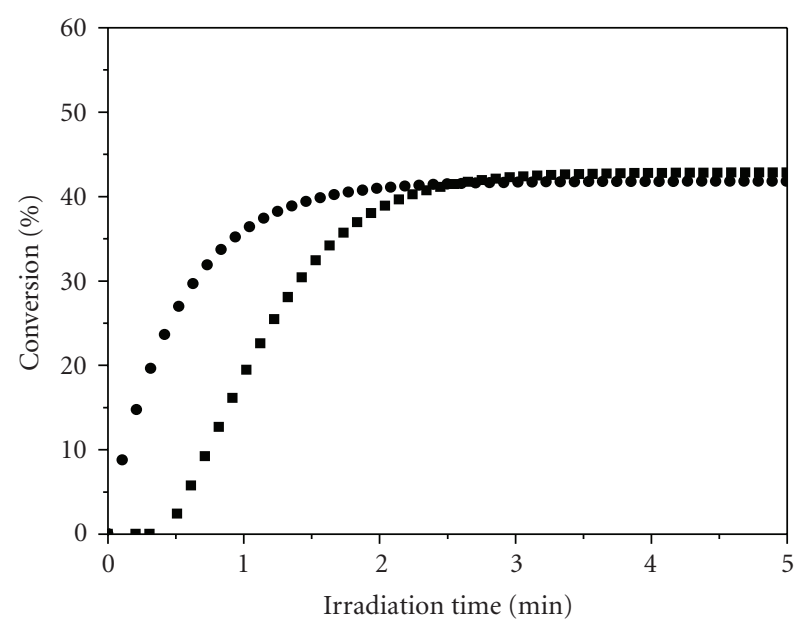

- NOFC-1

- $\mathrm{NOFC}-1+\mathrm{BPO}$

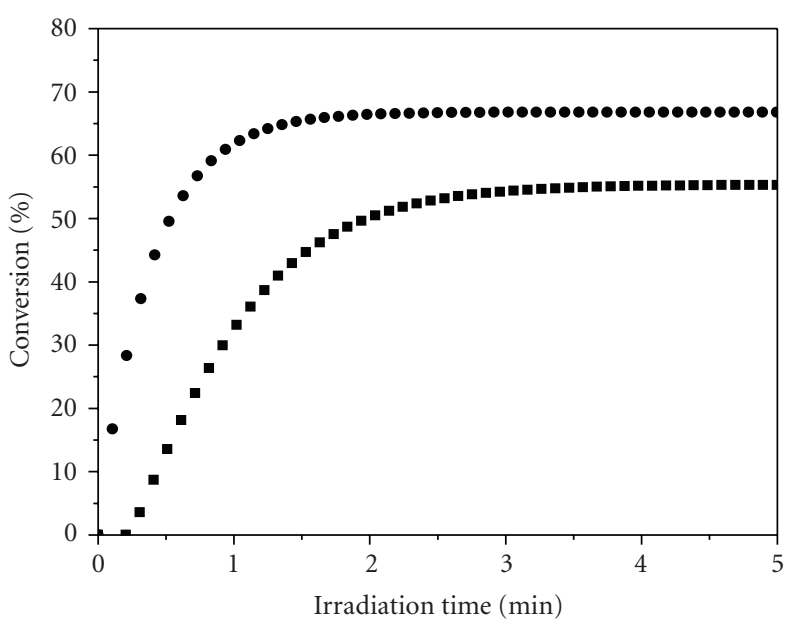

- NOFC-2

- $\mathrm{NOFC}-2+\mathrm{BPO}$

(a)

(b)

Figure 3: Conversion versus irradiation time plot of epoxy monomer ERL4221 initiated by $3.0 \mathrm{wt} \%$ photoinitiator with $3.0 \mathrm{wt} \%$ BPO as photosensitizer $\left(\mathrm{I}=80 \mathrm{~mW} / \mathrm{cm}^{2}\right)$.

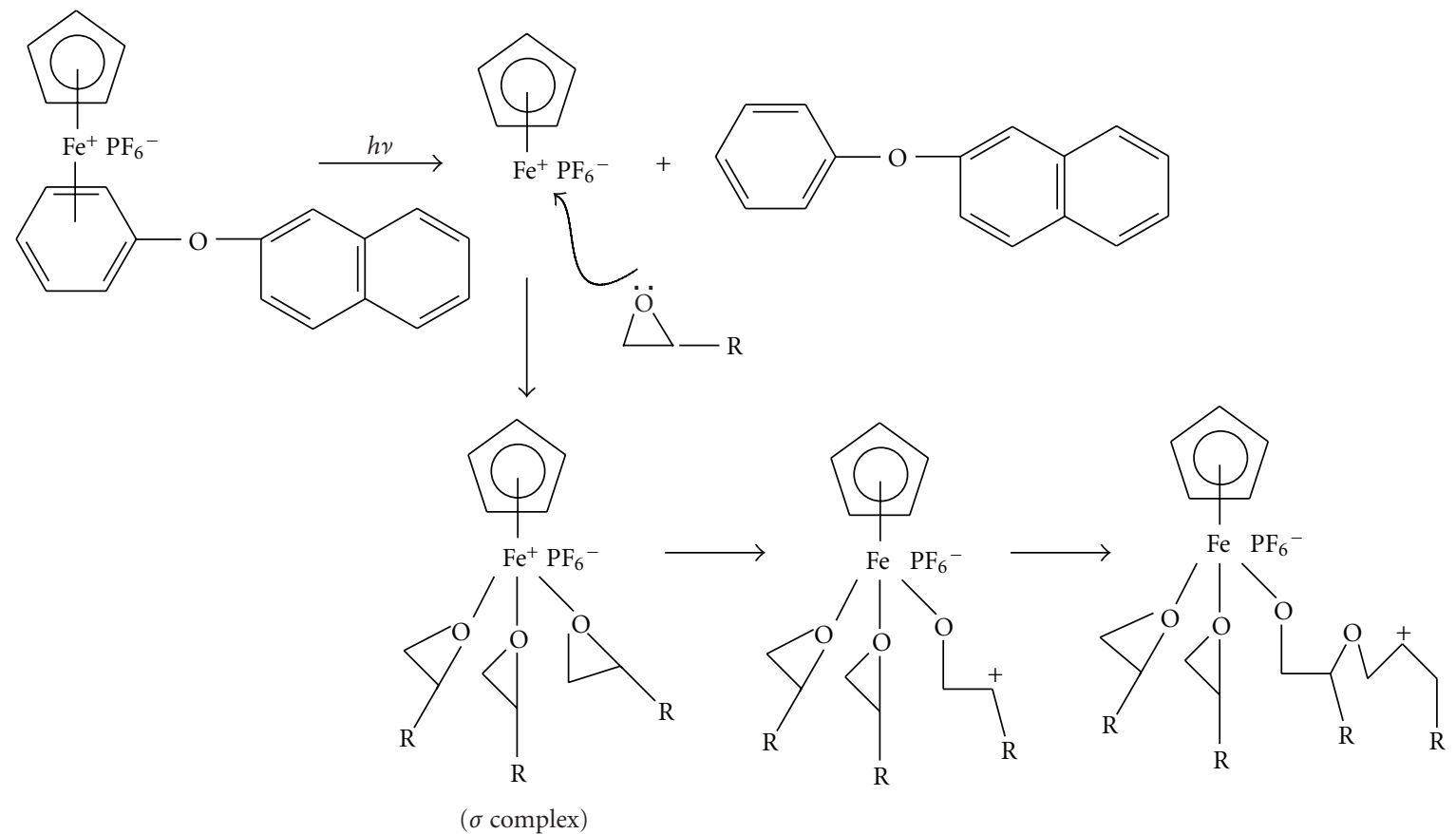

Scheme 3: Ring opening reaction of epoxide by an iron-arene complex.

exchange reaction between one ring of ferrocene and chlorobenzene according to the method of Nesmeyanov and coworkers [19]. It is known that metal moieties $\mathrm{FeCp}^{+}$can effectivly activate the halobenzene ring towards nucleophilic substitution reaction with phenoxides and alkoxides [2022 ]. This was accomplished through the use of a $1: 2$ molar ratio of the complex to naphthalenol, in the presence of excess $\mathrm{K}_{2} \mathrm{CO}_{3}$ in DMF. The mixtures were stirred under nitrogen at room temperature for 9 hours, and the products were isolated as yellow solids in very good yield (85-91\%). The synthesis of NOFC is summarized in Figure 2.

3.2. UV-Vis Absorption Spectra of NOFC-1 and NOFC2. UV-Vis absorption spectra of NOFC-1 and NOFC-2 in $\mathrm{CH}_{3} \mathrm{CN}$ are given in Figure 1. For comparison, the absorption spectra of I-261 are also presented. These spectra were recorded at identical molar concentration $\left(1 \times 10^{-4} \mathrm{M}\right.$ or $1 \times 10^{-3} \mathrm{M}$ ), enabling a direct comparison of absorbance. 


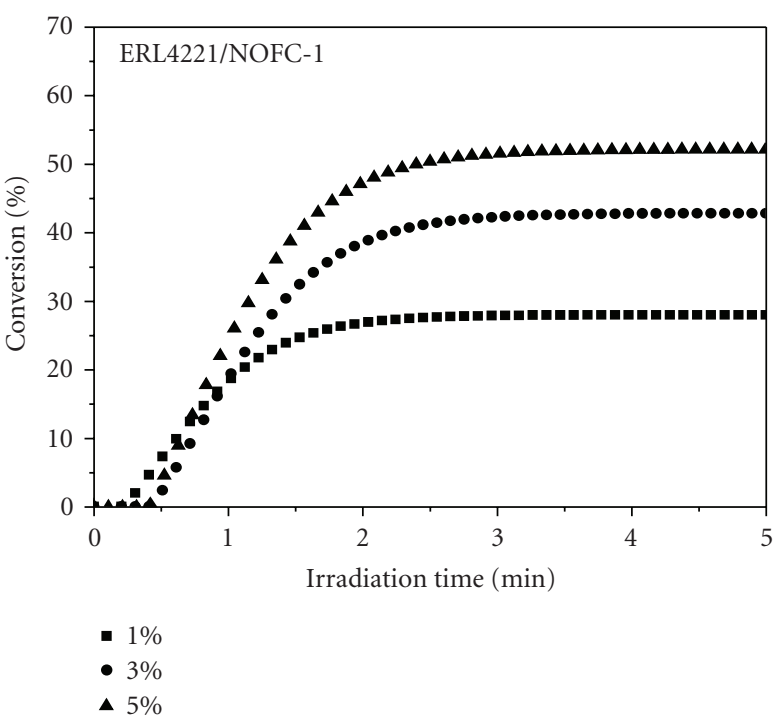

(a)

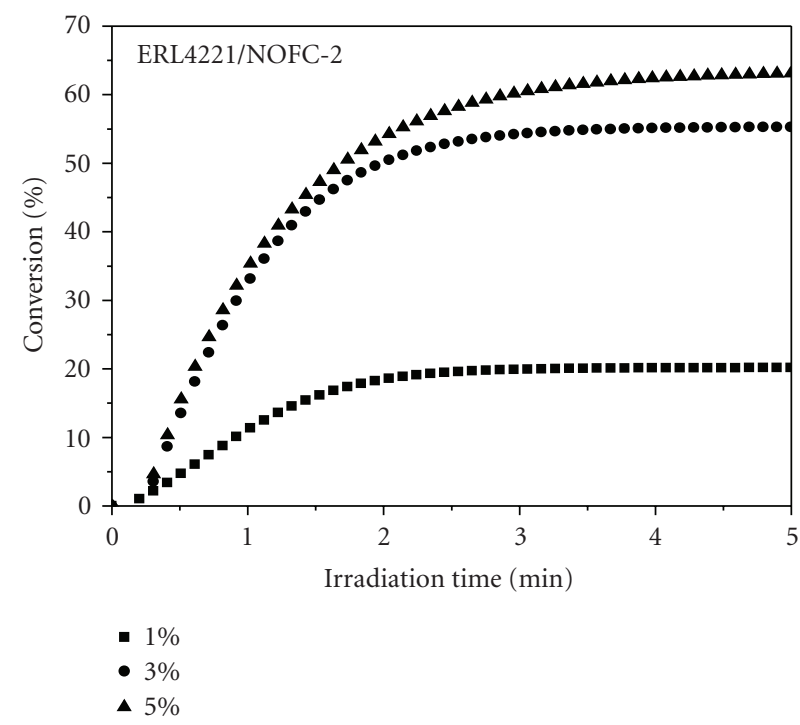

(b)

FIgURE 4: Effect of NOFC-1 and NOFC-2 concentration on the polymerization of ERL4221 $\left(\mathrm{I}=80 \mathrm{~mW} / \mathrm{cm}^{2}\right)$.

The UV-Vis absorption spectra of NOFC-1 and NOFC2 are similar to each other above $300 \mathrm{~nm}$ which makes them attractive as photoinitiators on irradiation with long wavelength UV light., and their absorption is stronger than that of I-261. The strongest absorption lies in the region of 200-300 $\mathrm{nm}$ which belongs to $\pi-\pi$ transition. The absorption at longer wavelength, ranging from $300 \mathrm{~nm}$ to $400 \mathrm{~nm}$, belongs to $\mathrm{d}$-d transition of iron arene complexes.

3.3. Photoinitiating Activity. In this investigation, RTIR spectroscopy was used to evaluate the efficiency of NOFC- 1 and NOFC-2 photoinitiators in the cationic polymerization of epoxy monomer ERL4221. ERL4221 is an important epoxy monomer because it is commercially available and has many applications for cationic photopolymerization.

The conversion versus time plots for the polymerization of ERL4221 carried out in the presence of ferrocenium salts NOFC-1, NOFC-2, and I-261 are shown in Figure 2. The results show that NOFC- 1 and NOFC-2 are capable of photoinitiating the cationic polymerization of epoxy monomer directly on irradiation with long wavelength UV light.

The comparative studies show that the polymerization rate of ERL4221 initiated by NOFC-1 and NOFC-2 is obviously higher than that of I-261. The final conversion yield of ERL4221 initiated by NOFC- 2 is the highest. The main reason was thought to be the more absorption of NOFC- 1 and NOFC-2 above $350 \mathrm{~nm}$ than that of I-261.

The photoinitiated polymerization of ERL4221 was carried out in the presence and absence of benzoyl peroxide (BPO) with NOFC-1 and NOFC-2 as the photoinitiators, and the results are shown in Figures 3 and 4. As shown in Figure 3 , there is an obvious increase in the photopolymerization rate of ERL4221 in the presence of $3.0 \mathrm{wt} \% \mathrm{BPO}$ comparing with in the absence of BPO.
The photoinitiated mechanism of epoxide monomer irradiated by ferrocenium salts is shown in Figure 3. With the loss of the arene ligand, an iron-based Lewis acid is generated from ferrocenium salts. Coordination of this ironbased Lewis acid with an epoxy monomer is followed by ringopening polymerization. It was commonly recognized that the photosensitizing role of peroxide $\mathrm{BPO}$ is oxidizing iron cation from two valence to a higher three valence and then enhance the rate of complex formation with epoxides.

The plots of conversion versus irradiation time of ERL4221 photoinitiated by NOFC-1 and NOFC-2 with different concentration are shown in Figure 4. The polymerization rate and final conversion increased with increase in NOFC concentration; because the higher the photoinitiator concentration is, the more the active species are produced during irradiation resulting in the higher rate of polymerization.

3.4. Thermal Stability. An important property of photoresists is the inherent thermal stability of the photoinitiator since pre- and post-exposure bakes can influence the physical state of the material. It is important because crosslinking of unexposed areas must be avoided in order to maximize the photoresist contrast. To assess this likelihood, DSC was employed to measure the onset of thermally initiated polymerization.

The samples of epoxy monomer ERL4221 with $1.0 \mathrm{wt} \%$ ferrocenium salts were evaluated over a temperature range between $50^{\circ} \mathrm{C}$ and $250^{\circ} \mathrm{C}$ at a heating rate of $10^{\circ} \mathrm{C} / \mathrm{min}$, respectively. From Figure 5 for DSC scan, these systems are stable under $160^{\circ} \mathrm{C}$. However, when the temperature reaches the onset temperature, the polymerization heat can be obtained without UV irradiation in these systems, and temperature in excess of the onset temperature should be avoided when processing these materials. 


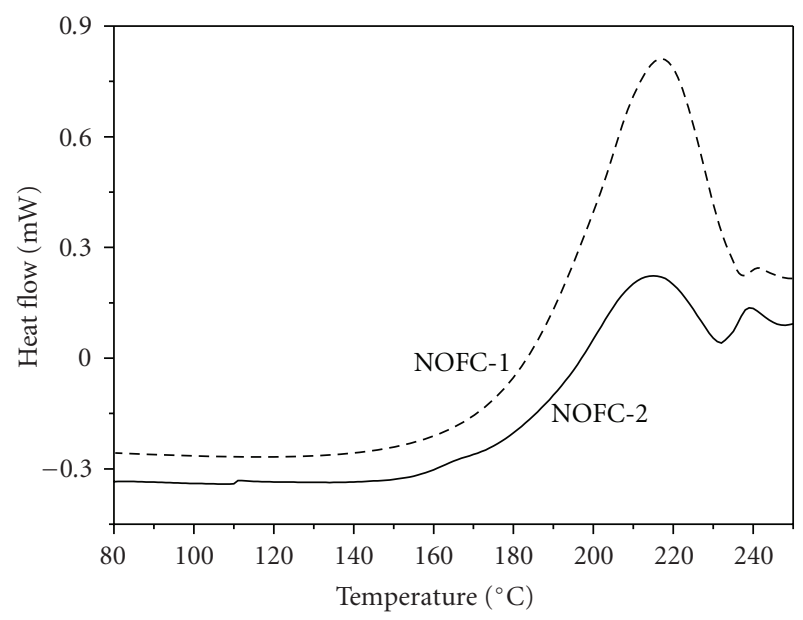

FIGURE 5: DSC scan of ERL-4221 with $1.0 \mathrm{wt} \%$ ferrocenium salts (heating rate $=10^{\circ} \mathrm{C} / \mathrm{min}$ static air).

\section{Conclusions}

Two new cationic photoinitiators, NOFC-1 and NOFC-2 have been synthesized. They can photoinitiate the cationic polymerization of ERL4221 directly on irradiation with high-pressure $\mathrm{Hg}$ light. Studies with real-time infrared spectroscopy have shown that NOFC-1 and NOFC-2 photoinitiators exhibit good efficiency in the polymerization of ERL4221 and BPO sensitizer is very effective in developing their photoinitiating activities. DSC studies have shown that NOFC-1 and NOFC-2 photoinitiators in epoxides possess good thermal stability under $160^{\circ} \mathrm{C}$ in the absence of light.

\section{Acknowledgment}

The authors wish to thank the financial support of National Natural Science Foundation of China (Project Grant no. 20676012).

\section{References}

[1] X. Y. Hong and H. B. Feng, "Cationic photopolymerization initiated with onium salts," Gaofenzi Tongbao, no. 1, pp. 1723, 1989.

[2] M. Sangermano and J. V. Crivello, "Visible and longwavelength cationic photopolymerization," in Proceedings of the ACS Symposium on Photoinitiated Polymerization, vol. 847 of ACS Symposium Series, pp. 242-252, 2003.

[3] R. Lazauskaite and J. V. Grazulevicius, "Cationic photopolymerization," in Handbook of Photochemistry and Photobiology, pp. 335-392, 2003.

[4] J. V. Crivello, "The discovery and development of onium salt cationic photoinitiators," Journal of Polymer Science, Part A, vol. 37, no. 23, pp. 4241-4254, 1999.

[5] J. V. Crivello and J. H. W. Lam, "Diaryliodonium salts: a new class of photoinitiators for cationic polymerization," Macromolecules, vol. 10, no. 6, pp. 1307-1315, 1977.

[6] J. V. Crivello and J. H. W. Lam, "Photoinitiated cationic polymerization by dialkyl-4-hydroxyphenylsulfonium salts,"
Journal of Polymer Science, Polymer Chemistry Edition, vol. 18, no. 3, pp. 1021-1034, 1980.

[7] J. V. Crivello and J. H. W. Lam, "A new preparation of triarylsulfonium and -selenonium salts via the copper(II)-catalyzed arylation of sulfides and selenides with diaryliodonium salts," Journal of Organic Chemistry, vol. 43, no. 15, pp. 3055-3058, 1978.

[8] J. L. Dektar and N. P. Hacker, "A new mechanism for photodecomposition and acid formation from triphenylsulphonium salts," Journal of the Chemical Society, Chemical Communications, no. 20, pp. 1591-1592, 1987.

[9] F. Castellanos, J. P. Fouassier, C. Priou, and J. Cavezzan, "Synthesis, reactivity, and properties of new diaryliodonium salts as photoinitiators for the cationic polymerization of epoxy silicones," Journal of Applied Polymer Science, vol. 60, no. 5, pp. 705-713, 1996.

[10] R. S. Davidson and J. W. Goodin, "Some studies on the photoinitiated cationic polymerisation of epoxides," European Polymer Journal, vol. 18, no. 7, pp. 589-595, 1982.

[11] R. J. Devoe, M. R. V. Sahyun, and E. Schmidt, "Electron transfer sensitized photolysis of onium salts," The Imaging Science Journal, vol. 33, no. 2, pp. 39-43, 1989.

[12] K. Meier and H. Zweifel, "Curable composition and it use," Journal of Imaging Science, vol. 30, no. 4, pp. 174-176, 1986.

[13] H. Zweifel and K. Meier, "Imaging with cationic organomftallic photoinitiators," American Chemical Society, Division of Polymer Chemistry, vol. 26, no. 2, pp. 347-348, 1985.

[14] T. Wang, P. Wan, and L. J. Ma, "Synthesis and characterization of alkoxy and phenoxy-substituted ferrocenium salt cationic photoinitiators," Chinese Journal of Chemical Engineering, vol. 14, no. 6, pp. 806-809, 2006.

[15] T. Wang and Z. H. Wang, "Cationic photopolymerization of epoxy systems initiated by cyclopentadien-iron-biphenyl hexafluorophosphate ([Cp-Fe-biphenyl $\left.]^{+} \mathrm{PF}_{6}{ }^{-}\right)$," Polymer Bulletin, vol. 53, no. 5-6, pp. 323-331, 2005.

[16] M. Degirmenci, G. Hizal, and Y. Yagci, "Synthesis and characterization of macrophotoinitiators of poly ( $\varepsilon$-caprolactone) and their use in block copolymerization," Macromolecules, vol. 35, no. 22, pp. 8265-8270, 2002.

[17] D. Mustafa, I. Cianga, and Y. Yagci, "Synthesis of well-defined polystyrene macrophotoinitiators by atom-transfer radical polymerization," Macromolecular Chemistry and Physics, vol. 203, no. 10-11, pp. 1279-1284, 2002.

[18] I. U. Khand, P. L. Pauson, and W. F. Watts, "Dienyl complexes of transition metals-II: addition of hydride to halogenstbstituted arenecyclopentadienyliron cations," Journal of the Chemical Society C, no. 17, pp. 2261-2265, 1968.

[19] A. N. Nesmeyanov, "Nuclear magnetic resonance spectra of arenecyclopentadienyliron compounds," Journal of Organometallic Chemistry, vol. 22, no. 3, pp. 689-696, 1970.

[20] A. J. Pearson and A. M. Gelormini, "Selective arylation of diols using arene-iron chemistry," The Journal of Organic Chemistry, vol. 60, no. 1, pp. 281-284, 1995.

[21] A. S. Abd-El-Aziz, Y. Lei, and C. R. De Denus, "Aliphatic diols in the synthesis of bis(cyclopentadienyliron) arene complexes," Polyhedron, vol. 14, no. 12, pp. 1585-1591, 1995.

[22] A. S. Abd-El-Aziz, D. C. Schriemer, and C. R. De Denus, "Bis(cyclopentadienyliron)arene complexes: a new route to the synthesis and functionalization of polyaromatic ethers," Organometallics, vol. 13, no. 1, pp. 374-384, 1994. 


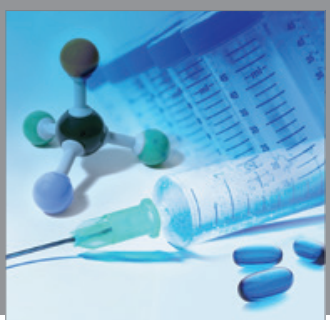

International Journal of

Medicinal Chemistry

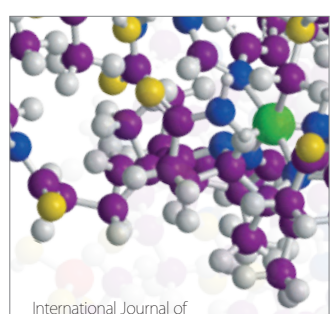

Carbohydrate Chemistry

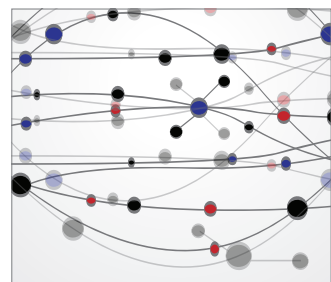

The Scientific World Journal
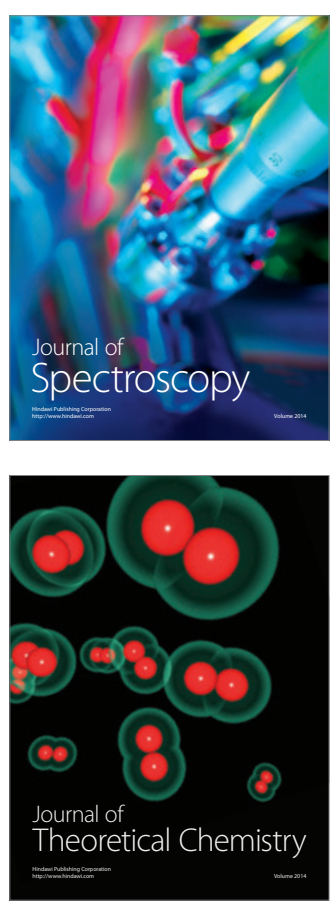
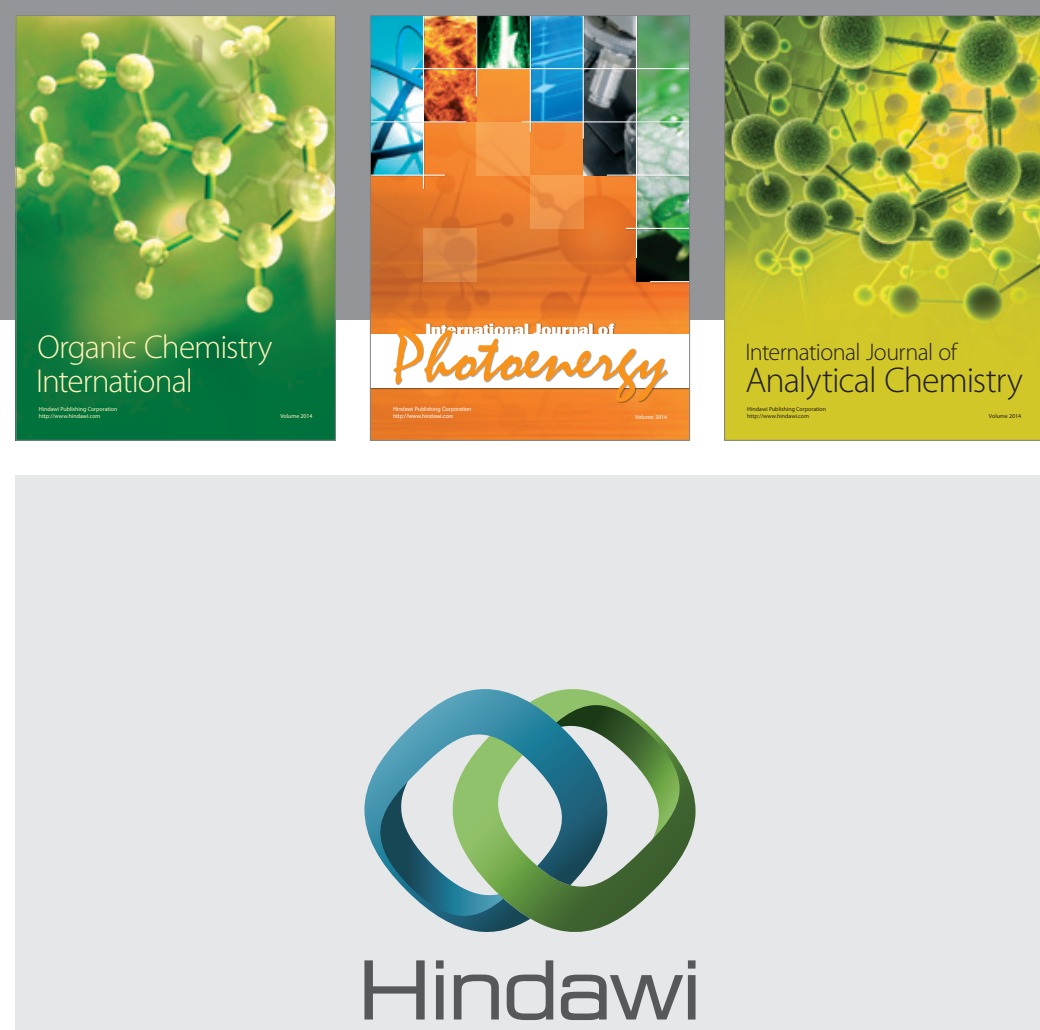

Submit your manuscripts at

http://www.hindawi.com
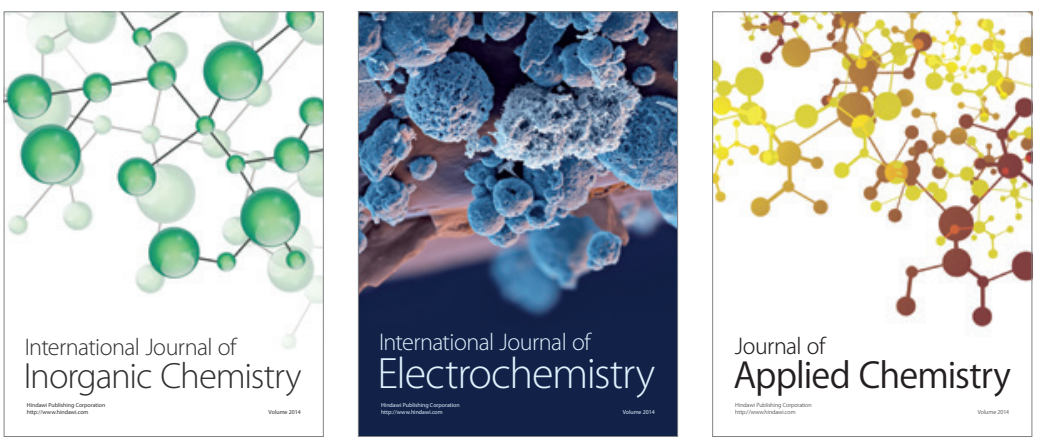

Journal of

Applied Chemistry
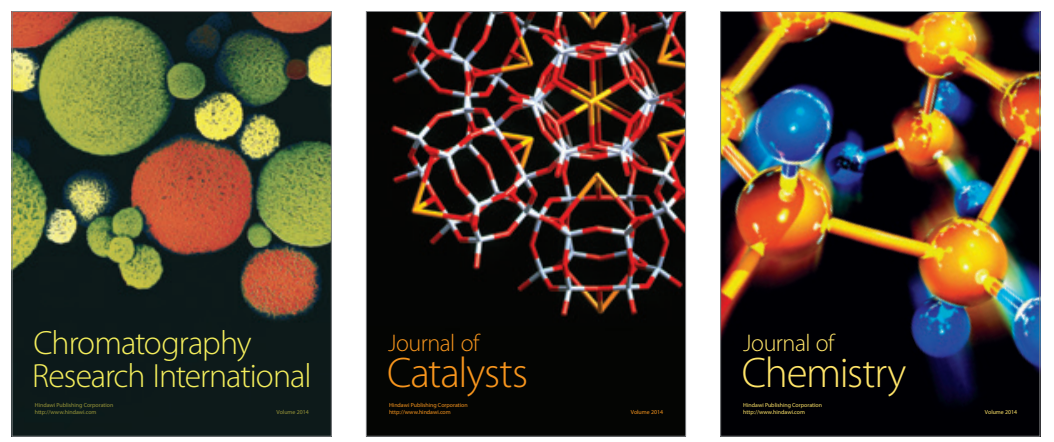
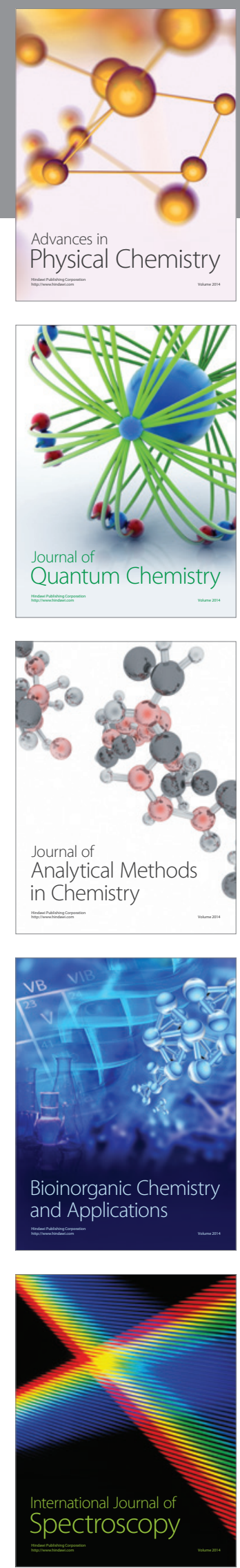